\title{
Direct reactions in/for astrophysics
}

\author{
Carlos Bertulani** \\ Department of Physics, University of Arizona, Tucson, AZ 85721 \\ E-mail: bertulani@physics.arizona.edu
}

Precise nuclear reaction rates are needed for a detailed description of the production of elements in primordial nucleosynthesis and during the hydrostatic burning of stars to constrain the astrophysical models. The relevant reactions are extremely difficult to measure directly in the laboratory at the small astrophysical energies. In recent years direct reactions methods have been developed and applied to extract low-energy astrophysical S-factors. The application of these methods requires a combination of experimental and theoretical efforts. This contribution focuses on the underlying reaction theories that have to be well understood in order to assess the precision and limitations of the various approaches.

International Symposium on Nuclear Astrophysics - Nuclei in the Cosmos - IX

25-30 June 2006

CERN

* Speaker.

${ }^{\dagger}$ I thank A. Mengoni and C. Spitaleri for beneficial discussions. This work was supported by the U.S. Department of Energy under Grant No. DE-FG02-04ER41338. 


\section{How difficult is nuclear astrophysics?}

Ongoing studies in nuclear astrophysics are focused on the opposite ends of the energy scale of nuclear reactions: (a) the very high and (b) the very low relative energies between the reacting nuclei. Projectiles with high bombarding energies produce nuclear matter at high densities and temperatures. One expects that matter produced in central nuclear collisions will undergo a phase transition and produce a quark-gluon plasma. One can thus reproduce conditions existing in the first seconds of the universe and also in the core of neutron stars. At the other end of the energy scale are the low energy reactions of importance for stellar evolution. Chains of nuclear reactions lead to complicated phenomena like nucleosynthesis, supernovae explosions, and energy production in stars.

In our Sun the reaction ${ }^{7} \mathrm{Be}(\mathrm{p}, \gamma)^{8} \mathrm{~B}$ plays a major role for the production of high energy neutrinos from the $\beta$-decay of ${ }^{8} \mathrm{~B}$. These neutrinos come directly from center of the Sun and are ideal probes of the sun's structure. John Bahcall frequently said that this was the most important reaction in nuclear astrophysics. Our knowledge about this reaction has improved considerably due to the appearance of radioactive beam facilities. The reaction ${ }^{12} \mathrm{C}(\alpha, \gamma){ }^{16} \mathrm{O}$ is extremely relevant for the fate of massive stars. It determines if the remnant of a supernova explosion becomes a black-hole or a neutron star. These two reactions are only two examples of a large number of reactions which are not yet known with the required accuracy needed in astrophysics.

Approximately half of all stable nuclei observed in nature in the heavy element region, $A>60$, are produced in the $\mathrm{r}$-process. This $\mathrm{r}$-process occurs in environments with large neutron densities which leads to neutron capture times much smaller than the beta-decay half-lives, $\tau_{\mathrm{n}} \ll \tau_{\beta}$. The most neutron-rich isotopes along the r-process path have lifetimes of less than one second; typically $10^{-2}$ to $10^{-1} \mathrm{~s}$. Cross sections for most of the nuclei involved are hard to measure experimentally. Sometimes, theoretical calculations of the capture cross sections as well as the beta-decay half-lives are the only source of the nuclear physics input for $\mathrm{r}$-process calculations.

Nucleosynthesis in stars is complicated by the presence of electrons. They screen the nuclear charges, therefore increasing the fusion probability by reducing the Coulomb repulsion. Evidently, the fusion cross sections measured in the laboratory have to be corrected by the electron screening when used in a stellar model. This is a purely theoretical problem as one can not reproduce the interior of stars in the laboratory.

A simpler screening mechanism occurs in laboratory experiments due to the bound atomic electrons in the nuclear targets. This case has been studied in great details experimentally, as one can control different charge states of the projectile+target system in the laboratory [1, 2, 3]. The experimental findings disagree systematically by a factor of two with theory. This is surprising as the theory for atomic screening in the laboratory relies on our basic knowledge of atomic physics. At very low energies one can use the simple adiabatic model in which the atomic electrons rapidly adjust their orbits to the relative motion between the nuclei prior to the fusion process. Energy conservation requires that the larger electronic binding (due to a larger charge of the combined system) leads to an increase of the relative motion between the nuclei, thus increasing the fusion cross section. As a matter of fact, this enhancement has been observed experimentally. The measured values are however not compatible with the adiabatic estimate [1, 2, 3]. Dynamical calculations have been performed, but they obviously cannot explain the discrepancy as they include atomic 
excitations and ionizations which reduce the energy available for fusion. Other small effects, like vacuum polarization, atomic and nuclear polarizabilities, relativistic effects, etc., have also been considered [4]. But the discrepancy between experiment and theory remains [4, 3].

A possible solution of the laboratory screening problem was proposed in refs. [5, 6]. Experimentalists often use the extrapolation of the Andersen-Ziegler tables [7] to obtain the average value of the projectile energy due to stopping in the target material. The stopping is due to ionization, electron-exchange, and other atomic mechanisms. However, the extrapolation is challenged by theoretical calculations which predict a lower stopping. Smaller stopping was indeed verified experimentally [3]. At very low energies, it is thought that the stopping mechanism is mainly due to electron exchange between projectile and target. This has been studied in ref. [8] in the simplest situation; proton+hydrogen collisions. The calculated stopping power was added to the nuclear stopping power mechanism, i.e. to the energy loss by the Coulomb repulsion between the nuclei. The obtained stopping power is proportional to $v^{\alpha}$, where $v$ is the projectile velocity and $\alpha=1.35$. The extrapolations from the Andersen-Ziegler table predict a smaller value of $\alpha$. Although this result seems to indicate the stopping mechanism as a possible reason for the laboratory screening problem, the theoretical calculations tend to disagree on the power of $v$ at low energy collisions.

For example, ref. [9] found $S \sim v_{p}^{3.34}$ for protons in the energy range of $4 \mathrm{keV}$ incident on helium targets. This is an even larger deviation from the extrapolations of the Andersen-Ziegler tables.

Another calculation of the stopping power in atomic $\mathrm{He}^{+}+\mathrm{He}$ collisions using the two-center molecular orbital basis was done in ref. [10]. The agreement with the data from ref. [9] at low energies is excellent. The agreement with the data disappears completely if the nuclear recoil is included. In fact, the unexpected "disappearance" of the nuclear recoil was also observed in ref. [11]. This seems to violate a basic principle of nature, as the nuclear recoil is due to the Coulomb repulsion between the projectile and the target atoms [7]. Much of what we know in this field now is due to the work of Claus Rolfs, Karlheinz Langanke, Noboru Takigawa, Kouichi Hagino, Baha Balantekin, and collaborators.

In the previous paragraphs I have described a few examples of typical problems in nuclear astrophysics. Now I discuss how direct reactions have been used to attempt solving part of these problems.

\section{Direct reactions in/for nuclear astrophysics}

The number of radioactive beam facilities are growing fast around the world. Some of these facilities use the fragmentation technique, with secondary beams in the energy range $E_{L a b} \approx 100$ $\mathrm{MeV} /$ nucleon. Examples are the facilities in GANIL/France, MSU/USA, RIKEN/Japan and GSI/Germany. In these facilities, direct reactions have become the main probe of nuclear structure of exotic nuclear species. In the following, I will present a short discussion of recent advances in direct reactions for nuclear astrophysics.

\subsection{Transfer reactions}

Transfer reactions are a well established tool to obtain spin, parities, energy, and spectroscopic factors of states in a nuclear system. Experimentally, $(d, p)$ reactions are popular due to the simplicity of the deuteron. Variations of this method have been proposed by several authors. Examples are 
the Trojan Horse Method (due to Gerhard Baur) and the Asymptotic Normalization Coefficients (due to Akram Mukhamedzhanov and Natasha Timofeyuk). They have been discussed by Bob Tribble in this conference. An advantage of using this technique over direct measurements is to avoid the treatment of the screening problem.

\subsection{Intermediate energy Coulomb excitation}

Before I go on and discuss the Coulomb dissociation method for nuclear astrophysics, I will discuss a few, and often neglected, effects in the theory of Coulomb excitation. In low-energy collisions the theory is very well understood [12]. A large number of small corrections are now well known in the theory and are necessary in order to analyze experiments on multiple excitation and reorientation effects. At the other end, the Coulomb excitation of relativistic heavy ions is characterized by straight-line trajectories with impact parameter $b$ larger than the sum of the radii of the two colliding nuclei. A derivation of relativistic electromagnetic excitation on this basis was performed by Aage Winther and Kurt Alder [14]. Later, it was shown that a quantum theory for relativistic Coulomb excitation leads to modifications of the semiclassical results [15]. In Refs. [17, 16] the inclusion of relativistic effects in semiclassical and quantum formulations of Coulomb excitation was fully clarified.

Recently, the importance of relativistic effects in Coulomb excitation of a projectile by a target with charge $Z_{2}$, followed by gamma-decay, in nuclear reactions at intermediate energies was studied in details. The Coulomb excitation cross section is given by

$$
\frac{d \sigma_{i \rightarrow f}}{d \Omega}=\left(\frac{d \sigma}{d \Omega}\right)_{\mathrm{el}} \frac{16 \pi^{2} Z_{2}^{2} e^{2}}{\hbar^{2}} \sum_{\pi \lambda \mu} \frac{B\left(\pi \lambda, I_{i} \rightarrow I_{f}\right)}{(2 \lambda+1)^{3}}|S(\pi \lambda, \mu)|^{2},
$$

where $B\left(\pi \lambda, I_{i} \rightarrow I_{f}\right)$ is the reduced transition probability of the projectile nucleus, $\pi \lambda=E 1, E 2$, $M 1, \ldots$ is the multipolarity of the excitation, and $\mu=-\lambda,-\lambda+1, \ldots, \lambda$.

The relativistic corrections to the Rutherford formula for $(d \sigma / d \Omega)_{\mathrm{el}}$ has been investigated in ref. [13]. It was shown that the scattering angle increases by up to $6 \%$ when relativistic corrections are included in nuclear collisions at $100 \mathrm{MeV} /$ nucleon. The effect on the elastic scattering cross section is even more drastic: up to $13 \%$ for center-of-mass scattering angles around 0-4 degrees.

The orbital integrals $S(\pi \lambda, \mu)$ contain the information about relativistic corrections. Inclusion of absorption effects in $S(\pi \lambda, \mu)$ due to the imaginary part of an optical nucleus-nucleus potential where worked out in ref. [16]. These orbital integrals depend on the Lorentz factor $\gamma=\left(1-v^{2} / c^{2}\right)^{-1 / 2}$, with $c$ being the speed of light, on the multipolarity $\pi \lambda \mu$, and on the adiabacity parameter $\xi(b)=\omega_{f i} b / \gamma v<1$, where $\omega_{f i}=\left(E_{f}-E_{i}\right) / \hbar$ is the excitation energy (in units of $\hbar$ ) and $b$ is the impact parameter.

A recent study in ref. [18] has shown that at $10 \mathrm{MeV} /$ nucleon the relativistic corrections are important only at the level of $1 \%$. At $500 \mathrm{MeV} /$ nucleon, the correct treatment of the recoil corrections is relevant on the level of $1 \%$. Thus the non-relativistic treatment of Coulomb excitation [12] can be safely used for energies below about $10 \mathrm{MeV} /$ nucleon and the relativistic treatment with a straight-line trajectory [14] is adequate above about $500 \mathrm{MeV} /$ nucleon. However at energies around 50 to $100 \mathrm{MeV} /$ nucleon, accelerator energies common to most radioactive beam facilities (MSU, RIKEN, GSI, GANIL), it is very important to use a correct treatment of recoil and relativistic effects, both kinematically and dynamically. At these energies, the corrections can add up to 
50\%. These effects were also shown in Ref. [17] for the case of excitation of giant resonances in collisions at intermediate energies.

A reliable extraction of useful nuclear properties, like the electromagnetic response (B(E2)values, $\gamma$-ray angular distribution, etc.) from Coulomb excitation experiments at intermediate energies requires a proper treatment of special relativity [18, 19]. The dynamical relativistic effects have often been neglected in the analysis of experiments elsewhere (see, e.g. [21]). The effect is highly non-linear, i.e. a $10 \%$ increase in the velocity might lead to a $50 \%$ increase (or decrease) of certain physical observables. A general review of the importance of the relativistic dynamical effects in intermediate energy collisions has been presented in ref. [22, 20].

\subsection{The Coulomb dissociation method}

I refer to the talk by Tohru Motobayshi for the latest experimental applications of the Coulomb dissociation method. The idea is quite simple. The (differential, or angle integrated) Coulomb breakup cross section for $a+A \longrightarrow b+c+A$ follows from eq. 2.1. It can be rewritten as

$$
\frac{d \sigma_{C}^{\pi \lambda}(\omega)}{d \Omega}=F^{\pi \lambda}(\omega ; \theta ; \phi) \cdot \sigma_{\gamma+a \rightarrow b+c}^{\pi \lambda}(\omega)
$$

where $\omega$ is the energy transferred from the relative motion to the breakup, and $\sigma_{\gamma+a \rightarrow b+c}^{\pi \lambda}(\omega)$ is the photo nuclear cross section for the multipolarity $\pi \lambda$ and photon energy $\omega$. The function $F^{\pi \lambda}$ depends on $\omega$, the relative motion energy, nuclear charges and radii, and the scattering angle $\Omega=(\theta, \phi) . F^{\pi \lambda}$ can be reliably calculated [15] for each multipolarity $\pi \lambda$. Time reversal allows one to deduce the radiative capture cross section $b+c \longrightarrow a+\gamma$ from $\sigma_{\gamma+a \rightarrow b+c}^{\pi \lambda}(\omega)$. This method was proposed by Baur, Bertulani and Rebel, ref. [23]. It has been tested successfully in a number of reactions of interest for astrophysics. The most celebrated case is the reaction ${ }^{7} \mathrm{Be}(\mathrm{p}, \gamma){ }^{8} \mathrm{~B}$, first studied by Motobayashi and collaborators [24], followed by numerous experiments in the last decade. For a recent discussion of the results obtained with the method, see e.g. ref. [25].

Motobayashi's experiment using the Coulomb dissociation method immediately raised the interest of John Bahcall because the cross section for the ${ }^{7} \mathrm{Be}(\mathrm{p}, \gamma)^{8} \mathrm{~B}$ is a crucial input in John's Standard Solar Model [26]. In the wake of Motobayshi's experiment, John sent me four handwritten letters asking for details of the method, to which I replied all by e-mail. Soon the discussion spread among several people. A curious article entitled "Electronic Battle over Solar Neutrinos", with a partial description of this discussion, was published in the Science magazine [27]. John was very happy that new methods had been found to access information on reactions of astrophysical interest. His quick interest for the subject was typical of his enthusiasm with new developments in science and, in particular, in nuclear astrophysics.

Eq. 2.2 is based on first-order perturbation theory. It also assumes that the nuclear contribution to the breakup is small, or that it can be separated under certain experimental conditions. The contribution of the nuclear breakup has been examined by several authors (see, e.g. [28]). ${ }^{8} \mathrm{~B}$ has a small proton separation energy $(\approx 140 \mathrm{keV})$. For such loosely-bound systems it had been shown that multiple-step, or higher-order effects, are important [29]. These effects occur by means of continuum-continuum transitions. Detailed studies of dynamic contributions to the breakup were explored in refs. [30, 31] and in several other publications which followed. The role of higher multipolarities (e.g., E2 contributions [32, 33, 34] in the reaction ${ }^{7} \mathrm{Be}(\mathrm{p}, \gamma)^{8} \mathrm{~B}$ ) and the coupling to 
high-lying states has also to be investigated carefully. In the later case, a recent work has shown that the influence of giant resonance states is small [35]. It is worthwhile mentioning that much of the theoretical advances in understanding the role of the nuclear interaction and of higher-order effects is due to the work of Stefan Typel, Angela Bonaccorso, Gerhard Baur, Daniel Baye, Felipe Canto, Radhey Shyam, and their collaborators.

\subsection{Charge exchange reactions}

During core collapse, temperatures and densities are high enough to ensure that nuclear statistical equilibrium is achieved. This means that for sufficiently low entropies, the matter composition is dominated by the nuclei with the highest binding energy for a given $Y_{e}$. Electron capture reduces $Y_{e}$, driving the nuclear composition to more neutron rich and heavier nuclei, including those with $N>40$, which dominate the matter composition for densities larger than a few $10^{10} \mathrm{~g} \mathrm{~cm}^{-3}$. As a consequence of the model applied in collapse simulations, electron capture on nuclei ceases at these densities and the capture is entirely due to free protons. To understand the whole process it is necessary to obtain Gamow-Teller matrix elements which are not accessible in beta-decay experiments. Many-body theoretical calculations are right now the only way to obtain the required matrix elements. This situation can be remedied experimentally by using charge-exchange reactions. Charge exchange reactions induced in $(\mathrm{p}, \mathrm{n})$ reactions are often used to obtain values of Gamow-Teller matrix elements, $B(G T)$, which cannot be extracted from beta-decay experiments. This approach relies on the similarity in spin-isospin space of charge-exchange reactions and $\beta$ decay operators. As a result of this similarity, the cross section $\sigma(\mathrm{p}, \mathrm{n})$ at small momentum transfer $q$ is closely proportional to $B(G T)$ for strong transitions [36]. Taddeucci's formula reads

$$
\frac{d \sigma}{d q}(q=0)=K N_{D}\left|J_{\sigma \tau}\right|^{2} B(\alpha)
$$

where $K$ is a kinematical factor, $N_{D}$ is a distortion factor (accounting for initial and final state interactions), $J_{\sigma \tau}$ is the Fourier transform of the effective nucleon-nucleon interaction, and $B(\alpha=$ $F, G T)$ is the reduced transition probability for non-spin-flip, $B(F)=\left(2 J_{i}+1\right)^{-1}\left|\left\langle f|| \sum_{k} \tau_{k}^{( \pm)}|| i\right\rangle\right|^{2}$, and spin-flip, $B(G T)=\left(2 J_{i}+1\right)^{-1}\left|\left\langle f|| \sum_{k} \sigma_{k} \tau_{k}^{( \pm)} \| i\right\rangle\right|^{2}$, transitions.

Taddeucci's formula, valid for one-step processes, was proven to work rather well for $(\mathrm{p}, \mathrm{n})$ reactions (with a few exceptions). For heavy ion reactions the formula might not work so well. This has been investigated in refs. [37, 38, 39]. In ref. [37] it was shown that multistep processes involving the physical exchange of a proton and a neutron can still play an important role up to bombarding energies of $100 \mathrm{MeV} /$ nucleon. Refs. [38, 39] use the isospin terms of the effective interaction to show that deviations from the Taddeucci formula are common under many circumstances. As shown in ref. [40], for important GT transitions whose strength are a small fraction of the sum rule the direct relationship between $\sigma(\mathrm{p}, \mathrm{n})$ and $B(G T)$ values also fails to exist. Similar discrepancies have been observed [41] for reactions on some odd-A nuclei including ${ }^{13} \mathrm{C},{ }^{15} \mathrm{~N}$, ${ }^{35} \mathrm{Cl}$, and ${ }^{39} \mathrm{~K}$ and for charge-exchange induced by heavy ions [39, 42]. In summary, it is still an open question if Taddeucci's formula is valid in general.

Undoubtedly, charge-exchange reactions such as (p,n), $\left({ }^{3} \mathrm{He}, \mathrm{t}\right)$ and heavy-ion reactions (A,A \pm 1$)$ can provide information on the $B(F)$ and $B(G T)$ values needed for astrophysical purposes. This will certainly be one of the major research areas in radioactive beam facilities. This project needs 
to include an active collaboration for the reaction-theory part of this method. A very promising project lead by Remco Zegers and Sam Austin at the NSCL/MSU, using charge-exchange reactions for astrophysical purposes, is currently under way.

\subsection{Knock-out reactions}

Exotic nuclei are the raw materials for the synthesis of the heavier elements in the Universe, and are of considerable importance in nuclear astrophysics. Modern shell-model calculations are also now able to include the effects of residual interactions between pairs of nucleons, using forces that reproduce the measured masses, charge radii and low-lying excited states of a large number of nuclei. For very exotic nuclei the small additional stability that comes with the filling of a particular orbital can have profound effects upon their existence as bound systems, their lifetimes and structures. Thus, verifications of the ordering, spacing and the occupancy of orbitals are essential in assessing how exotic nuclei evolve in the presence of large neutron or proton imbalance and our ability to predict these theoretically. Such spectroscopy of the states of individual nucleons in short-lived nuclei uses direct nuclear reactions. The relentless work of P. Gregers Hansen on knockout reactions was one of the most beautiful chapters of modern nuclear physics. Quoting Gregers [43]: "Neutron saturated nuclei are the closest one can get to having a neutron star in the laboratory. The study of drip-line nuclei has progressed remarkably by observing nuclear reactions caused by radioactive fragments."

Single-nucleon knockout reactions with heavy ions, at intermediate energies and in inverse kinematics, have become a specific and quantitative tool for studying single-particle occupancies and correlation effects in the nuclear shell model. The experiments observe reactions in which fast, mass $A$, projectiles collide peripherally with a light nuclear target producing residues with mass $(A-1)$. The final state of the target and that of the struck nucleon are not observed, but instead the energy of the final state of the residue can be identified by measuring coincidences with decay gamma-rays emitted in flight.

The early interest in knockout reactions came from studies of nuclear halo states, for which the narrow momentum distributions of the core fragments in a qualitative way revealed the large spatial extension of the halo wave function. It was shown by Bertulani and McVoy [44] that the longitudinal component of the momentum (taken along the beam or $z$ direction) gave the most accurate information on the intrinsic properties of the halo and that it was insensitive to details of the collision and the size of the target. In contrast to this, the transverse distributions of the core are significantly broadened by diffractive effects and by Coulomb scattering. For experiments that observe the nucleon produced in elastic breakup, the transverse momentum is entirely dominated by diffractive effects, as illustrated [45] by the angular distribution of the neutrons from the reaction ${ }^{9} \mathrm{Be}\left({ }^{11} \mathrm{Be},{ }^{10} \mathrm{Be}+\mathrm{n}\right) \mathrm{X}$. In this case, the width of the transverse momentum distribution reflects essentially the size of the target.

To understand the measured longitudinal momentum distributions it is necessary to take into account that a heavy-ion knockout reaction, being surface-dominated, can only sample the external part of the nucleon wave function. The magnitude of the reaction cross section is determined by the part of the wave function that is accessed, and the shape of the momentum distribution reflects the momentum content in this part. Calculations [46, 47, 48] based on a sharp-surface strong-absorption ("black-disk") model could account for the observed longitudinal momentum 
distributions and also, approximately, for the absolute cross sections. This approach is confirmed in the, more accurate work of Bertulani and Hansen [49], which extends the theory to include the general dependence of the differential cross section on the momentum vector. The work of Mahir Hussein and Kirk McVoy [50], Angela Bonaccorso and David Brink [51], Kai Hencken, Henning Esbensen and George Bertsch [48, 52] and of Jim Alkhalili and Jeff Tostevin [53, 54, 55, 56, 57] were crucial for the development of theoretical tools for knock-out reactions.

\section{Reconciling nuclear structure with nuclear reactions}

Many reactions of interest for nuclear astrophysics involve nuclei close to the dripline. To describe these reactions, a knowledge of the structure in the continuum is a crucial feature. Recent work by Alexander Volya and Vladimir Zelevinsky [58], Nicolas Michel, Witek Nazarewicz, Marek Ploszajczak, Karim Bennaceur [59], and collaborators, are paving the way toward a microscopic understanding of the many-body continuum. One basic theoretical problem is to what extent we know the form of the effective interactions for threshold states. It is also hopeless that these methods can be accurate in describing high-lying states in the continuum. In particular, it is not worthwhile to pursue this approach to describe direct nuclear reactions.

One immediate goal can be achieved in the coming years by using the Resonating Group Method (RGM) or the Generator Coordinate Method (GCM). These are a set of coupled integrodifferential equations of the form

$$
\sum_{\alpha^{\prime}} \int d^{3} r^{\prime}\left[H_{\alpha \alpha^{\prime}}^{A B}\left(\mathbf{r}, \mathbf{r}^{\prime}\right)-E N_{\alpha \alpha^{\prime}}^{A B}\left(\mathbf{r}, \mathbf{r}^{\prime}\right)\right] g_{\alpha^{\prime}}\left(\mathbf{r}^{\prime}\right)=0
$$

where $H_{\alpha \alpha^{\prime}}^{A B}\left(\mathbf{r}, \mathbf{r}^{\prime}\right)=\left\langle\Psi_{A}(\alpha, \mathbf{r})|H| \Psi_{B}\left(\alpha^{\prime}, \mathbf{r}^{\prime}\right)\right\rangle$ and $N_{\alpha \alpha^{\prime}}^{A B}\left(\mathbf{r}, \mathbf{r}^{\prime}\right)=\left\langle\Psi_{A}(\alpha, \mathbf{r}) \mid \Psi_{B}\left(\alpha^{\prime}, \mathbf{r}^{\prime}\right)\right\rangle$. In these equations $H$ is the Hamiltonian for the system of two nuclei (A and B) with the energy $E, \Psi_{A, B}$ is the wavefunction of nucleus $\mathrm{A}$ (and B), and $g_{\alpha}(\mathbf{r})$ is a function to be found by numerical solution of eq. 3.1, which describes the relative motion of $\mathrm{A}$ and $\mathrm{B}$ in channel $\alpha$. Full antisymmetrization between nucleons of A and B are implicit. Modern nuclear shell-model calculations, including the No-Core-Shell-Model (NCSM) are able to provide the wavefunctions $\Psi_{A, B}$ for light nuclei. But the Hamiltonian involves an effective interaction in the continuum between the clusters A and B. It is not possible to obtain this effective interaction within the NCSM presently. Great progress in the microscopic theory of nuclear reactions has been obtained by Pierre Descouvemont and Daniel Baye. More applications of the RGM method using NCSM wavefunctions was presented by Christian Forssen in this conference.

Overlap integrals of the type $I_{A a}(r)=\left\langle\Psi_{A-a} \mid \Psi_{A}\right\rangle$ for bound states has been calculated by Petr Navratil [60] within the NCSM. This is one of the inputs necessary to calculate S-factors for radiative capture, $S_{\alpha} \sim\left|\left\langle g_{\alpha}\left|H_{E M}\right| I_{A a}\right\rangle\right|^{2}$, where $H_{E M}$ is a corresponding electromagnetic operator. The left-hand side of this equation is to be obtained by solving eq. 3.1. For some cases, in particular for the $\mathrm{p}+{ }^{7} \mathrm{Be}$ reaction, the distortion caused by the microscopic structure of the cluster does not seem to be crucial to obtain the wavefunction in the continuum. The wavefunction is often obtained by means of a potential model. The NCSM overlap integrals, $I_{A a}$, can also be corrected to reproduce the right asymptotics [61], given by $I_{A a}(r) \propto W_{-\eta, l+1 / 2}\left(2 k_{0} r\right)$, where $\eta$ is the Sommerfeld parameter, $l$ the angular momentum, $k_{0}=\sqrt{2 \mu E_{0}} / \hbar$ with $\mu$ the reduced mass and $E_{0}$ the separation energy. 
A step in the direction of reconciling structure and reactions for the practical purpose of obtaining astrophysical S-factors, along the lines described in the previous paragraph, was obtained in ref. [61, 62]. The wavefunctions obtained in this way were shown to reproduce very well the momentum distributions in knockout reactions of the type ${ }^{8} \mathrm{~B}+A \longrightarrow{ }^{7} \mathrm{Be}+X$ obtained in experiments at MSU and GSI facilities. The astrophysical S-factor for the reaction ${ }^{7} \mathrm{Be}(\mathrm{p}, \gamma){ }^{8} \mathrm{~B}$ was also calculated and excellent agreement was found with the experimental data in both direct and indirect measurements [61, 62]. The low- and high-energy slopes of the S-factor obtained with the NCSM is well described by the fit

$$
S_{17}(E)=(22.109 \mathrm{eV} . \mathrm{b}) \frac{1+5.30 E+1.65 E^{2}+0.857 E^{3}}{1+E / 0.1375},
$$

where $\mathrm{E}$ is the relative energy (in $\mathrm{MeV}$ ) of $\mathrm{p}+{ }^{7} \mathrm{Be}$ in their center-of-mass. This equation corresponds to a Padé approximant of the $\mathrm{S}$-factor. A subthreshold pole due to the binding energy of ${ }^{8} \mathrm{~B}$ is responsible for the denominator [63, 64].

\section{Future}

Extremely exciting experimental results on direct reactions in/for nuclear astrophysics will be produced in the future. New radioactive beam facilities will be constructed around the world. Among the several proposed experiments, I mention the R3B (contact person: Thomas Aumann) and the ELISE (contact person: Haik Simon) projects, both at the future FAIR facility in GSI. The first project will use radioactive beams and direct reactions to obtain the nuclear physics input for astrophysics. The ELISE experimental setup will use electrons scattered off radioactive nuclei. These experiments will explore an unknown world of studies with nuclei far from stability which play an important role in our universe.

The US needs urgently a new radioactive beam facility, fully dedicated to the physics of radioactive nuclei. Without competing facilities worldwide, observational and theoretical astrophysics will never be able to constrain numerous models used to understand our universe. And without new inputs and constraints set by nuclear physics, astrophysics would slowly become a "no-man's land" (or "all-you-can-eat") science.

\section{References}

[1] H.J. Assenbaum, K. Langanke and C. Rolfs, Z. Phys. A327 (1987) 461.

[2] C. Rolfs and E. Somorjai, Nucl. Inst. Meth. B99 (1995) 297.

[3] C. Rolfs, Prog. Part. Nucl. Phys. 46 (2001) 23.

[4] A.B. Balantekin, C.A. Bertulani, M.S. Hussein, Nucl. Phys. A627 (1997) 324.

[5] K. Langanke, T.D. Shoppa, C.A. Barnes and C. Rolfs, Phys. Lett. B369, 211 (1996).

[6] J.M. Bang, L.S. Ferreira, E. Maglione, and J.M. Hansteen, Phys. Rev. C53, R18 (1996).

[7] H. Andersen and J.F. Ziegler, "The Stopping and Ranges of Ions in Matter", Pergamon, NY, 1977.

[8] C.A. Bertulani and D.T. de Paula, Phys. Rev. C62 (2000) 045802. 
[9] R. Golser and D. Semrad, Phys. Rev. Lett. 14, 1831 (1991).

[10] C.A. Bertulani, Phys. Let. B 585 (2004) 35.

[11] A. Formicola et al., Eur. Phys. J. A8, 443 (2000).

[12] K. Alder and A. Winther, Electromagnetic Excitation, North-Holland, Amsterdam, 1975.

[13] C.E.Aguiar, A.N.F.Aleixo and C.A.Bertulani, Phys. Rev. C 42, 2180 (1990).

[14] A. Winther and K. Alder, Nucl. Phys. A 319 (1979) 518.

[15] C.A.Bertulani and G.Baur, Phys. Reports 163 (1988) 299.

[16] C.A. Bertulani and A.M. Nathan, Nucl. Phys. A 554 (1993) 158.

[17] A.N.F. Aleixo and C.A. Bertulani, Nucl. Phys. A 505 (1989) 448.

[18] C.A. Bertulani, A. Stuchbery, T. Mertzimekis and A. Davies, Phys. Rev. C 68, 044609 (2003).

[19] C.A. Bertulani, C.M. Campbell, and T. Glasmacher, Comput. Phys. Commun. 152, 317 (2003).

[20] C.A.Bertulani, Phys. Rev. Lett. 94, 072701 (2005).

[21] T. Glasmacher, Nucl. Phys. A 693 (2001) 90.

[22] C.A. Bertulani, Proceedings of Workshop on "Reaction Mechanisms for Rare Isotope Beams" Michigan State University March 9-12,2005, arXiv.org:nucl-th/0505015.

[23] G. Baur, C. Bertulani and H. Rebel, Nucl. Phys. A459 (1986) 188.

[24] T. Motobayashi, et al., Phys. Rev. Lett. 73 (1994) 2680.

[25] H. Esbensen, G.F. Bertsch, and K. Snover, Phys. Rev. Lett. 94,042502 (2005); Moshe Gai, Phys. Rev. Lett. 96, 159201 (2006); H. Esbensen, G.F. Bertsch, and K. Snover, Phys. Rev. Lett. 94, 042502 (2005).

[26] J.N. Bahcall,“Neutrino Astrophysics”, Cambridge University Press, 1989.

[27] Gary Taubes, Science, Volume 266, Issue 5188, pp. 1157.

[28] C.A. Bertulani and M. Gai, Nucl. Phys. A636 (1998) 227.

[29] C.A.Bertulani and L.F.Canto, Nucl. Phys. A 539 (1992) 163

[30] G. Baur, C.A. Bertulani and D.M. Kalassa, Nucl. Phys. A550 (1992) 527.

[31] G.F. Bertsch and C.A. Bertulani, Nucl. Phys. A556 (1993) 136; Phys. Rev. C49 (1994) 2834; H. Esbensen, G.F. Bertsch and C.A. Bertulani, Nucl. Phys. A581 (1995) 107.

[32] C.A. Bertulani, Phys. Rev. C49 (1994) 2688; Nucl. Phys. A587 (1995) 318; Z. Phys. A356 (1996) 293.

[33] M. Gai and C.A. Bertulani, Phys. Rev. C52 (1995) 1706; Nucl. Phys. A636 (1998) 227.

[34] H. Esbensen and G. Bertsch, Phys. Rev. Lett. A359 (1995) 13; Nucl. Phys. A600 (1996) 37.

[35] C.A. Bertulani, Phys. Lett. B3 (2002) 205.

[36] T.N. Tadeucci et al., Nucl. Phys. A469 (1987) 125.

[37] H. Lenske, H.H. Wolter and H.G. Bohlen, C.A. Bertulani, Phys. Rev. Lett. 62, 1457 (1989).

[38] C.A. Bertulani, Nucl. Phys. A 554 (1993) 493. 
[39] C.A. Bertulani and P. Lotti, Phys. Lett. B 402 (1997) 237; C.A. Bertulani and D. Dolci, Nucl. Phys. A674 (2000) 527.

[40] S.M. Austin, N. Anantaraman and W.G. Love, Phys. Rev. Lett. 73 (1994) 30.

[41] J.W. Watson et al., Phys. Rev. Lett. 55 (1985) 1369.

[42] M. Steiner, et al., Phys. Rev. Lett. 76 (1996) 26.

[43] P. Gregers Hansen, Nature 334, 194 (1988).

[44] C. A. Bertulani and K. W. McVoy, Phys. Rev. C 46, 2638 (1992).

[45] R. Anne, et al., Nucl. Phys. A 575 (1994) 125.

[46] P. G. Hansen, A. S. Jensen and B. Jonson, Annu. Rev. Nucl. Part. Sci. 45, 505 (1995).

[47] P. G. Hansen, Phys. Rev. Lett. 77, 1016 (1996).

[48] H. Esbensen, Phys. Rev. C 53, 2007 (1996).

[49] C. A. Bertulani and P. G. Hansen, Phys. Rev. C 70, 034609 (2004).

[50] M. S. Hussein and K. W. McVoy, Nucl. Phys. A445 (1985) 123.

[51] A. Bonaccorso and D. M. Brink, Phys. Rev. C 44, 1559 (1991).

[52] K. Hencken, G. Bertsch and H. Esbensen, Phys. Rev. C 54 (1996) 3043.

[53] J. S. Al-Khalili, J. A. Tostevin and I. J. Thompson, Phys. Rev. C 54, 1843 (1996).

[54] J. A. Tostevin and J. S. Al-Khalili, Nucl. Phys. A 616, 418c (1997).

[55] J. A. Tostevin, J. Phys. G 25, 735 (1999).

[56] J. A. Tostevin, Nucl. Phys. A 682, 320c (2001).

[57] P. G. Hansen and J. A. Tostevin, Annu. Rev. Nucl. Part. Sci. 53, 219 (2003).

[58] A. Volya and V. Zelevinsky, Phys. Rev. Lett. 94, 052501 (2005)

[59] N. Michel, W. Nazarewicz and M. Ploszajczak, Phys. Rev. C 70, 064313 (2004).

[60] P. Navratil, Phys. Rev. C. 70, 054324 (2004).

[61] P. Navratil, C.A. Bertulani, and E. Caurier, Phys. Lett. B 634 (2006) 191.

[62] P. Navratil, C.A. Bertulani, and E. Caurier, Phys. Rev. C, in press.

[63] B. K. Jennings, S. Karataglidis, and T. D. Shoppa, Phys. Rev. C 58, 579 (1998).

[64] R. D. Williams and S. E. Koonin, Phys. Rev. C 23, 2773 (1981). 